\title{
L'impulsion bibliothécaire de la révolution scientifique : livres et réseaux autour de Johannes Kepler
}

Patrick J. Boner, Miguel A. Granada et Édouard Mehl

\section{(2) OpenEdition}

Journals

Édition électronique

URL : https://journals.openedition.org/rbnu/1598

DOI : 10.4000/rbnu. 1598

ISSN : 2679-6104

Éditeur

Bibliothèque nationale et universitaire de Strasbourg

\section{Édition imprimée}

Date de publication : 1 novembre 2014

Pagination : 10-21

ISBN : 9782859230548

ISSN : 2109-2761

\section{Référence électronique}

Patrick J. Boner, Miguel A. Granada et Édouard Mehl, « L'impulsion bibliothécaire de la révolution scientifique : livres et réseaux autour de Johannes Kepler », La Revue de la BNU [En ligne], 10 | 2014, mis en ligne le 01 novembre 2014, consulté le 17 mai 2021. URL : http://journals.openedition.org/ rbnu/1598; DOI : https://doi.org/10.4000/rbnu. 1598 


\section{loANNIS KePPIERI}

Mathematici Cofarci, hanc Imaginem.

\section{ARGENTORATENSI BIBLOTHECE.} Confecr.

\section{MatTHLAS Berneccervs.} $\mathrm{Kal}$. lanuar. Anno Chr.

M. DC. XXVII.
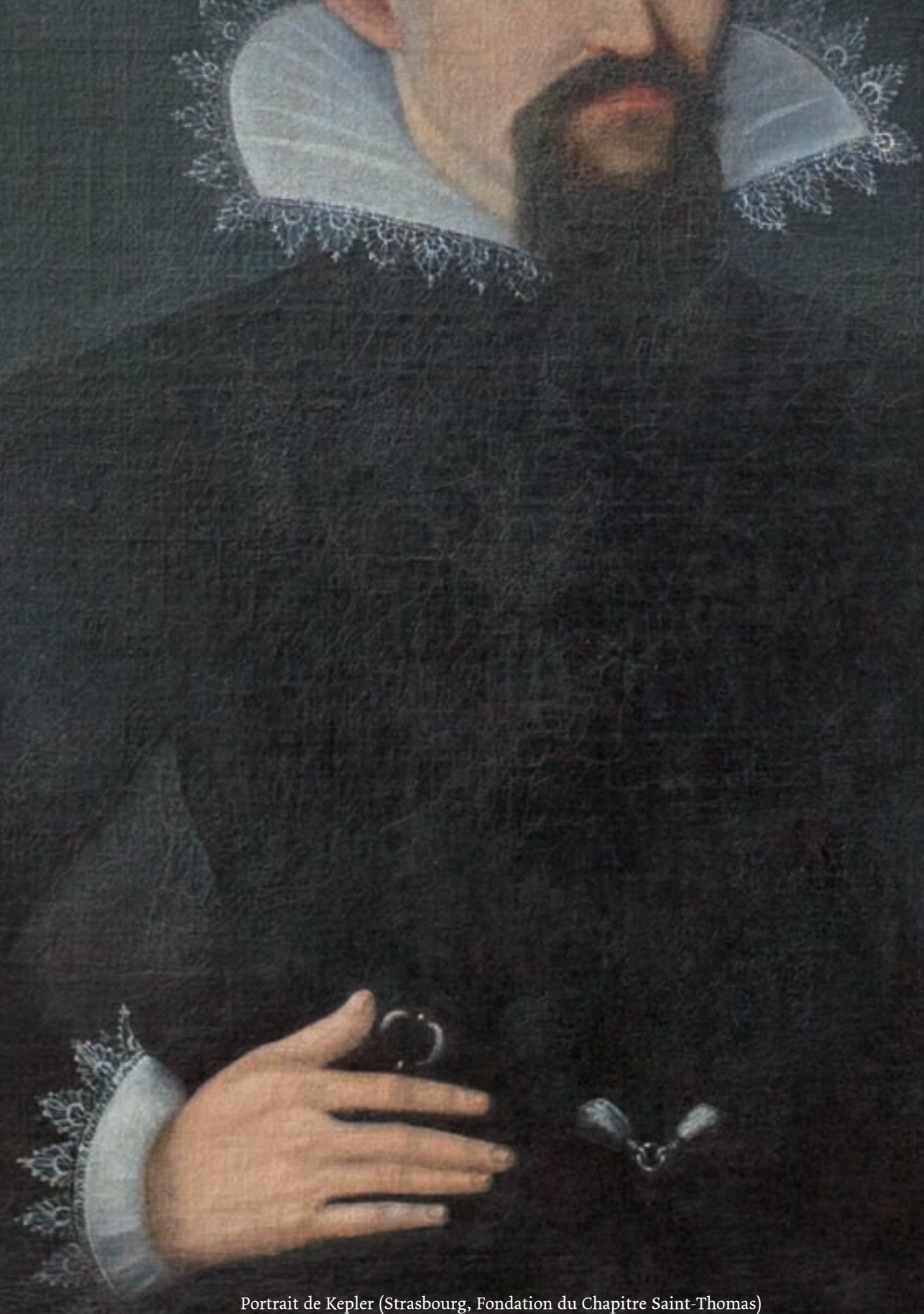


\section{L'impulsion bibliothécaire de la révolution scientifique : livres et réseaux autour de Johannes Kepler}

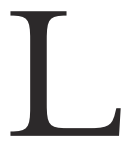

a tendance positiviste et scientiste qui dominait en histoire des sciences il y a encore peu de temps a pu accréditer la vision biaisée d'une révolution scientifique liée à l'émancipation vis-à-vis de la lecture et de la culture, comme si l'étude des phénomènes et le déchiffrement d'un " grand livre de la nature ", écrit en langage mathématique (Galilée), exigeaient qu'on se détournât des livres et de leurs théories branlantes, supposés constituer autant d'obstacles à l'appréhension directe du réel. On a même prétendu que l'adhésion au nouveau système du monde, copernicien, n'était pas liée à la diffusion du De revolutionibus orbium colestium (1543) - un livre que, selon une formule provocante d'Arthur Koestler, personne n'aurait lu. Justice a été faite de cette mythologie hasardeuse ${ }^{1}$, et l'on voudrait, à l'appui de cette réévaluation, montrer ici quel a été le rôle des livres et des bibliothèques dans la constitution d'un réseau transrégional de culture scientifique sans lequel l'œuvre monumentale de Kepler (1571-1630) n'eût jamais vu le jour.

\section{Le Corpus mathematicum de Conrad Dasypodius (1530-1600)}

Conrad Dasypodius (Rauchfuss), professeur de mathématiques à la Haute École de Strasbourg, est surtout connu du grand public pour être l'artisan de l'horloge astronomique de la cathédrale, bâtie sur ses instructions entre 1571 et 1574 . Comme l'a montré la monographie consacrée à cette merveilleuse machine ${ }^{2}$, la prouesse mécanique et l'inventivité technique de cette réalisation sont indissociables des recherches que le scholarque mène parallèlement sur l'histoire des mathématiques - ou plus exactement d'une science mathématique universelle embrassant aussi bien l'astronomie, l'optique, la mécanique, que toutes les sciences spéciales qui leur sont subordonnées -, et du vaste projet éditorial d'un corpus mathematicum allant des Éléments d'Euclide à une collection de textes grecs inédits, dont Dasypodius s'est procuré les copies à grands frais.

Ce projet est similaire, et en fait étroitement apparenté à celui que mène l'humaniste italien Federico Commandino (1509-1575), avec qui Dasypodius est en relation épistolaire, et l'on ne doit donc pas s'étonner de trouver des recoupements dans l'activité éditoriale des deux mathématiciens : ils exhument notamment l'œuvre astronomique et mathématique d'Aristarque de Samos (Des grandeurs du soleil et de la lune, et de leurs distances réciproques), qu'on cite comme le précurseur de Copernic, ou les Collections de Pappus d'Alexandrie, texte fondamental pour le développement moderne du concept d'analyse ${ }^{3}$. A quoi il faut ajouter, dans le projet du corpus dasypodien, maints traités anciens sur la musique ${ }^{4}$ - de ceux-là même que le chancelier Georg Herwart von Hohenburg fera connaître à Kepler, comme on le verra plus loin ${ }^{5}$-, ainsi qu'un ensemble d'astrologie gréco-arabe centré sur la réception et le commentaire du Tetrabiblos ptoléméen.

Un tel monument exigeait des moyens, notamment financiers, que Dasypodius pensait obtenir grâce au patronage du landgrave Moritz von Hessen-Kassel (1572-1632). Moritz, dit "le Savant ", portait l'héritage politique et scientifique de son père, Wilhelm IV von Hessen-Kassel (1532-1592), protecteur des sciences, correspondant de Tycho Brahe, fondateur du premier observatoire astronomique européen à Kassel, et à qui Dasypodius avait déjà fait allégeance en lui adressant un texte qu'il n'avait pas lui-même écrit, mais qu'il avait publié à Strasbourg en 1568, les Hypotyposes orbium cœlestium - premier manuel 
scolaire d'astronomie intégrant le fruit des travaux de la nouvelle astronomie copernicienne à l'enseignement traditionnel des Theorica planetarum hérité de Regiomontanus et Peurbach ${ }^{6}$. Las ! accablé de soucis personnels et familiaux, Dasypodius s'éteint en 1600 sans avoir pu mener à bien son grand projet éditorial. La majeure partie de sa bibliothèque échoit au strasbourgeois Matthias Bernegger $^{7}$; mais le fonds Bernegger, racheté par l'Université de Strasbourg à sa mort (1640), disparaît dans l'incendie du 24 août 1870. Ce sont donc, paradoxalement, les prises de guerre suédoises de la guerre de Trente Ans qui auront permis de sauver quelques miettes de ce trésor, et c'est à la bibliothèque de l'Université d'Uppsala que se trouvent aujourd'hui quelques restes de ce fonds scientifique exceptionnel.

Avec ce naufrage, certaines questions restent sans réponse, comme celle du sens à donner à la présence énigmatique du portrait de Copernic sur les panneaux de l'horloge astronomique, alors qu'on serait bien en peine de trouver dans l'œuvre du scholarque une déclaration qui ressemble de près ou de loin à une adhésion formelle au système héliocentrique, telle qu'on peut la trouver, par exemple, chez son contemporain Michael Mästlin (1550-1631) ${ }^{8}$. Cependant, le réseau académique et scientifique auquel appartient Dasypodius à Strasbourg est beaucoup moins compartimenté qu'on ne peut le penser. Comme l'attestent de nouveaux documents, d'une importance décisive, Dasypodius, au moment de lancer la construction de l'horloge, attendait que son " ami " Ioachim Rheticus (1514-1574), seul et unique disciple de Copernic et principal maître d'œuvre de la publication du De revolutionibus, lui livre les manuscrits anciens qu'il avait alors en sa possession ${ }^{9}$. Par ailleurs l'implication - active ou passive, il est difficile de le dire - de Rheticus dans le réseau scientifique strasbourgeois ne s'arrête pas là : le pupille de Wittenberg (qui n'y est plus en odeur de sainteté) a également des liens avec un ancien compagnon d'études, devenu professeur à Strasbourg, Michael Toxites, qui s'emploie à la tâche de publier les œuvres inédites de Paracelse, et compte alors sur la collaboration de son infortuné condisciple, admirateur déclaré du médecin errant ${ }^{10}$. Là aussi, la mort de Rheticus en 1574 est probablement ce qui interrompt l'exécution de ce dessein.

Ces éléments de contexte sont nécessaires pour mieux appréhender la grande controverse qui va, une dizaine d'années plus tard, opposer à Strasbourg un médecin paracelsien qui ne cache pas ses sympathies schwenckfeldiennes, Helisæus Röslin (1546-1616), et un jeune mathématicien qui a fait ses armes auprès de Conrad Dasypodius, avant d'obtenir à Prague la position de "mathématicien impérial ", Nicolas Reimers Ursus (1551-1600) ${ }^{11}$.

\section{La fondation d'une bibliothèque scientifique : Georg Herwart von Hohenburg (1553-1622)}

Depuis Munich, Johann Georg Herwart von Hohenburg, chancelier de Bavière, a joué un rôle central dans le milieu où évoluent ces différents protagonistes, en fondant, avec la bibliothèque dont il avait la charge dans cette ville, un lieu de savoir richement "fourni, aux frais du duc de Bavière, en livres et manuscrits grecs et hébreux " ${ }^{12}$. Pour mieux en assurer la diffusion et la publicité, Herwart a notamment édité le catalogue des manuscrits grecs. "Quiconque, élevé dans la foi catholique, désire contribuer à la République des lettres aura soin de veiller à leur intégrité ", plaide-t-il dans sa préface ${ }^{13}$. En dépit de ce que cette déclaration peut suggérer, Herwart n'a jamais laissé les querelles confessionnelles envahir et limiter le champ de ses relations, comme le montrent sa monumentale correspondance avec le luthérien Kepler, ou encore ses échanges également très suivis avec l'Alsacien hétérodoxe Helisaeus Röslin ${ }^{14}$. À travers un réseau épistolaire embrassant toutes sortes de sujets et de correspondants en Europe, Herwart s'est employé à créer une nouvelle chronologie, soit - rien de moins - une histoire mondiale fondée sur l'étude des textes anciens et vérifiée par le calcul astronomique.

Tandis que la bibliothèque de la Cour servait, grâce à ses efforts, à plusieurs autres de ses condisciples ${ }^{15}$, Herwart s'est appuyé sur les extraordinaires ressources offertes par ses collections pour bâtir cette nouvelle chronologie. Élément central au cœur de ce projet : Herwart ambitionnait de recréer le cours de l'Histoire en conformité avec un décompte exact et complet des anciennes éclipses. Tel était " l'exact et vrai décompte du temps " (vera et exacta temporis ratio) qu'il annonçait fièrement à ses contemporains ${ }^{16}$. Mais comment Herwart pouvait-il maîtriser la lune, dont les mouvements avaient confondu tant d'astronomes depuis tant de siècles ${ }^{17}$ ? Le défi n'avait pas échappé à son contemporain Johann Appenzeller : "Qui affirme que le mouvement de la lune est constamment irrégulier ne dit rien de nouveau ", écrit-il, "à moins qu'il ne montre en même temps quelle est la grandeur de cette variation et comment elle peut être ramenée à la règle ${ }^{18}$. Appenzeller affirme que les éclipses de l'époque du Christ ne sont pas moins difficiles à déterminer avec exactitude que celles des autres époques, et voit un désaccord " énorme " ${ }^{19}$ entre les éclipses qui précèdent et celles qui succèdent à l'époque historique du Christ. Herwart se savait incapable de résoudre ce problème par lui-même ; c'est ainsi qu'il fit appel à Tycho Brahe et à ses tables lunaires. 


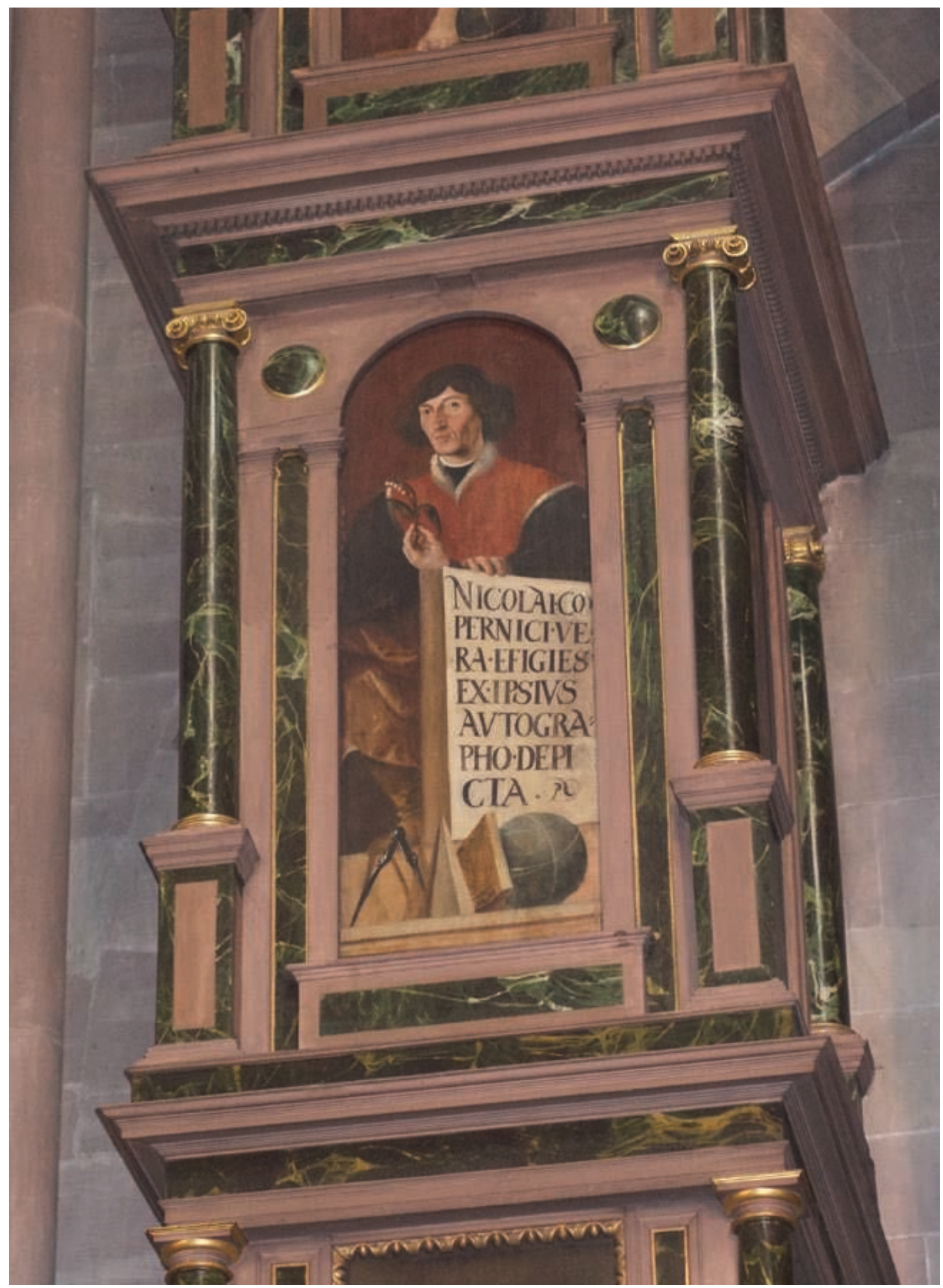

Portrait de Nicolas Copernic par Tobias Stimmer (Strasbourg, horloge astronomique de la cathédrale) 
Herwart pouvait-il exploiter les ressources de la nouvelle astronomie pour retracer le cours des cieux jusque dans le plus lointain passé ? Toujours est-il qu'il conçut de grandes espérances à l'annonce, par le pétulant Tycho Brahe, d'une nouvelle " restauration " de l'astronomie, et il lui fit part de son intention d'appliquer les mêmes principes à la chronologie. Tycho ayant prédit les éclipses du $16^{\mathrm{e}}$ siècle avec «beaucoup plus d'exactitude que toutes les tables connues ", Herwart s'avisa d'employer la même méthode pour déchiffrer les éclipses contemporaines du Christ et immédiatement antérieures. L'une d'entre elles retint particulièrement son attention. Il ne s'agissait en fait ni d'une éclipse lunaire, ni d'une éclipse solaire, mais d'une occultation de Vénus qui, " selon le rapport d'un ancien poète romain ", était apparue " au début de l'an 4 avant JésusChrist ". Herwart présumait que Mercure avait rattrapé Vénus et avait dissimulé l'étoile du matin juste au moment où, avant l'aube, elle aurait dû paraître aux regards. Son soupçon ne fit que croître en constatant que, selon les Tables pruténiques (établies d'après les calculs de Copernic, et dont Tychoconteste l'exactitude), les deux planètes différaient en latitude de plus de $2^{\circ} 44^{\prime}$. Herwart plaça donc tous ses espoirs en Tycho. "J’aurais désespéré ", lui écrivait-il, "si je n'avais découvert que tu avais relevé des latitudes très différentes, en particulier pour Vénus et Mercure, que celles que donnent les [autres] calculs ". Herwart implora donc le secours de Tycho, en échange de " toute espèce de service " ${ }^{20}$, dont la mise à disposition d'un choix d'ouvrages mathématiques en provenance de la bibliothèque bavaroise ${ }^{21}$.

Brahe ne se fit guère prier. Il commença par demander cinquante ouvrages au chancelier de Bavière, puis en réclama six autres en provenance de la Foire de Francfort ${ }^{22}$. Mais le prince des astronomes ne s'en tint pas là : en échange de ses services, Brahe demanda au chancelier de lui faire parvenir les manuscrits de Cyprianus Leovitius (1514-1574) conservés à Augsbourg. Tycho avait en effet souvenir de les avoir consultés lors de son séjour dans cette ville, dont il avait pu fréquenter la " vaste bibliothèque " appartenant aux Fugger. Il se trouve qu'Herwart était originaire d'une puissante famille d'Augsbourg, et y avait conservé toute son influence. Il y retournait régulièrement et y collaborait notamment avec son cousin Marcus Welser
(1558-1614), personnalité influente dans les cercles académiques et scientifiques, qui allait bientôt se trouver au cœur du débat entre Galilée et les Jésuites sur les taches solaires. En guise d'ultime faveur, Tycho demanda donc à Herwart un inventaire complet de la bibliothèque d'Augsbourg, et « spécialement des titres mathématiques et philosophiques ${ }^{23}$.

En échange de ces libéralités, Tycho remplit sa promesse de régler cette affaire d'éclipse. Avant d'entreprendre l'analyse du cas, il s'enquit de la fiabilité de ce témoignage littéraire. L'auteur n'avait-il pas usé de sa licence poétique pour falsifier la description des planètes ? L'astronome danois présumait, à bon droit, que le métier d'un poète est plutôt de faire des rimes que de tenir des observations exactes : "Même les historiens, qui veulent être plus véridiques, n'ont pu atteindre à la rigoureuse exactitude des cieux ${ }^{24}$. Pour preuve de leur impéritie, Tycho invoquait leur fréquente méprise et la confusion entre les comètes et de simples manifestations météorologiques. Tandis que Tycho avait montré que les comètes " accomplissent leur course dans les cieux ", il était arrivé depuis des siècles que les historiens aient pris pour des comètes ce qui n'était que de simples phénomènes météorologiques. Tycho donnait d'autres raisons de douter de l'occultation de Vénus : non seulement Mercure est trop petite pour recouvrir l'étoile du matin, mais en cas de conjonction, il estimait que " la lumière de Mercure se fût ajoutée à celle de Vénus, et celle-ci n'eût fait qu'augmenter au lieu de diminuer ${ }^{25}$. Au grand dam d'Herwart, les autres spécialistes consultés devaient faire part de réserves du même genre.

En effet, lorsque le chancelier revint à la charge à propos de cette même éclipse, le prince des astronomes transféra la requête à Joseph Scaliger (1540-1609) et promit à Herwart de lui tenir copie de sa réponse. Maître incontesté de la chronologie, Scaliger ne daigna pas prendre l'affaire au sérieux, et prétendit qu' " un homme aussi savant " ne pouvait se laisser séduire par de telles fausses idées que par défaut de mémoire, ou par un excès de confiance en un " auteur peu fiable " : "Qui donc est ce poète romain, sans nom ni prénom, et où diable a-t-il trouvé que cette éclipse aurait eu lieu en l'an 4 avant Jésus-Christ ? ${ }^{26}$ ". Mais plutôt que de blâmer le chancelier de Bavière, Scaliger 


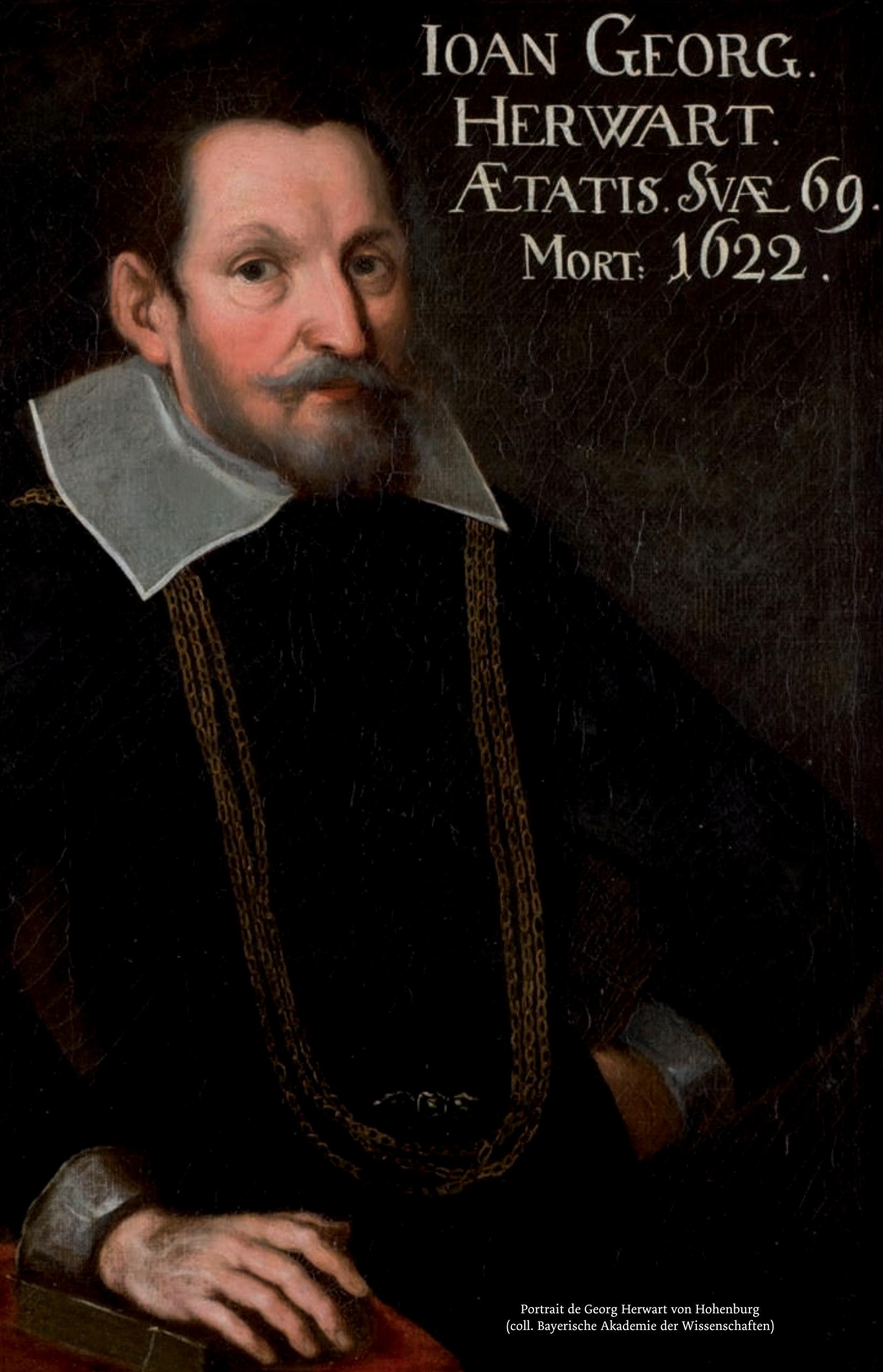




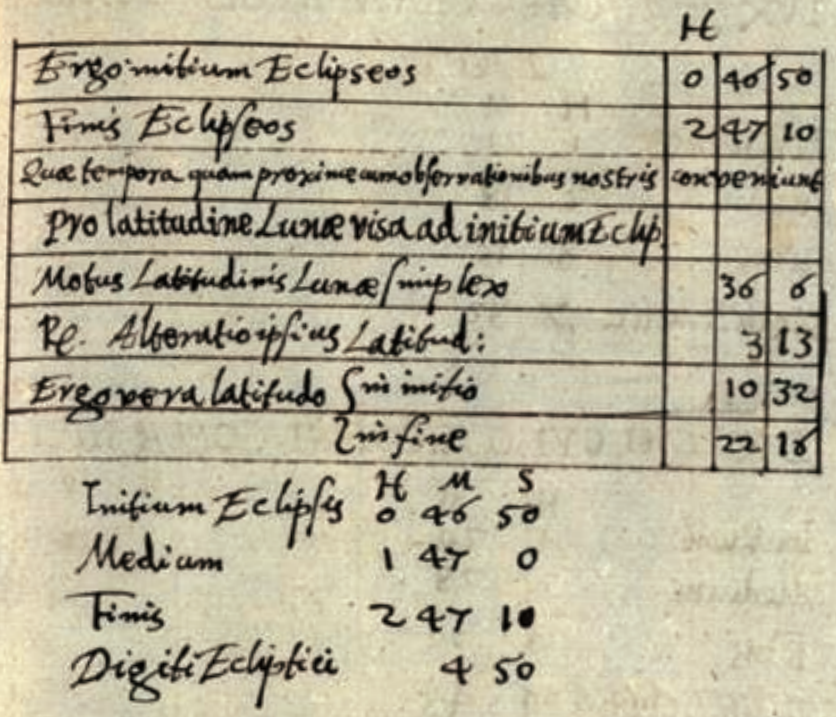

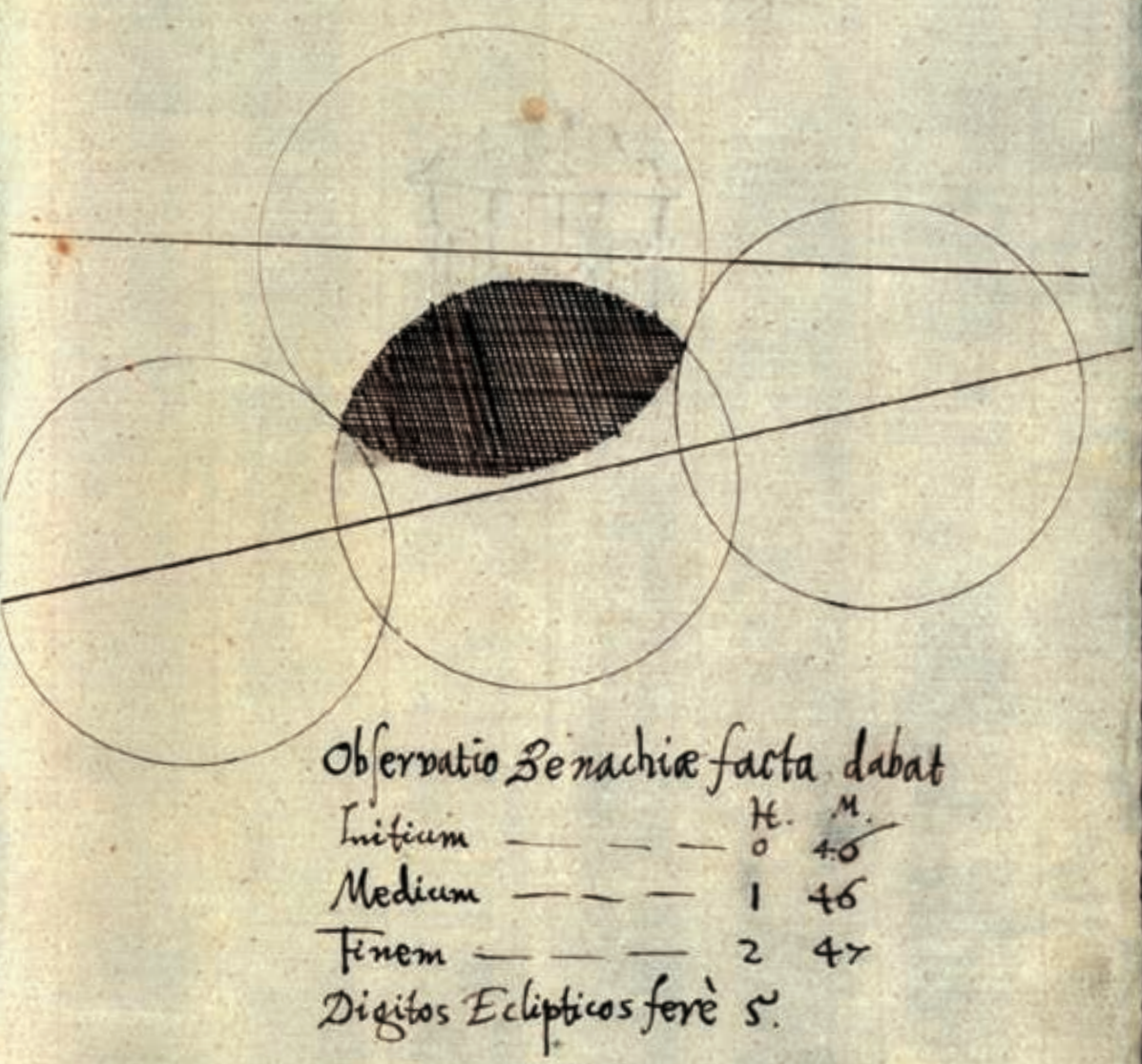

、 
présuma qu'il avait été trop occupé par ses fonctions pour consulter de meilleures sources. Son jugement devait arriver à Munich juste après que Tycho avait exprimé ses propres réserves. "Même si ce poète dit la vérité", dit finalement celui-ci au chancelier, " je croirais plutôt que Vénus a disparu derrière des émanations aériennes ou je ne sais quel phénomène météorologique qui a rendu l'air plus brillant [qu'elle] ${ }^{27}$ ". La mort brutale de Tycho ayant interrompu ces échanges, c'est vers le jeune mathématicien Kepler que se tourna Herwart, pour lui ouvrir les trésors de sa bibliothèque, en échange de son expertise scientifique ${ }^{28}$.

\section{Les manuscrits de la révolution astronomique : Helisaeus Röslin, Michael Mästlin, Wilhelm Schickard}

Propriété d'Herwart von Hohenburg, le codex 592 de l'Universitätsbibliothek de Munich contient cinq lettres adressées en 1597 au chancelier bavarois par Helisaeus Röslin ${ }^{29}$. Dans ces lettres, Röslin, médecin souabe résidant en Alsace, exprimait son jugement, assez sévère, sur un ouvrage de Johannes Kepler (le Mysterium cosmographicum récemment publié). Mais il répondait aussi à une requête du chancelier concernant la chronologie et en profitait pour s'attaquer violemment à l'astronome impérial Nicolas Reimers Ursus. Röslin y accusait Ursus d'avoir plagié avec son Chronotheatrum (Prague, 1597) sa propre chronologie, restée inédite faute de patronage, mais qu'Ursus avait eu l'occasion de lire lors de son séjour à Strasbourg en 1588. Arrivant tout juste de Kassel, Ursus avait donné une série de leçons à l'Académie de Strasbourg, et y avait soutenu des thèses sur un nouveau système cosmologique géohéliocentrique - thèses aussitôt publiées dans son Fundamentum astronomicum (Strasbourg, Bernard Jobin, 1588).

Cet événement devait immédiatement déclencher une " guerre " fameuse avec Tycho Brahe pour la priorité dans la découverte du système géohéliocentrique ${ }^{30}$. Au cours de ces mois, entre 1588 et 1589 , Röslin écrivit à son ami Michael Mästlin (1550-1631), professeur de mathématiques à l'Université de Tübingen, une série de lettres décrivant l'activité d'Ursus à Strasbourg et l'élaboration de sa propre version du système géohéliocentrique, dont l'édition devait toutefois attendre jusqu'à la publication tardive de son De opere Dei creationis seu de mundo hypotheses (Frankfurt, 1597). Dans sa correspondance avec Herwart, Röslin témoignait de son intention de répondre aux accusations diffamatoires du De astronomicis hypothesibus d'Ursus (Prague, 1596), tout en revendiquant son indépendance dans l'élaboration de son système géohéliocentrique, ce dont aurait dû attester la publication de sa correspondance avec Mästlin. Röslin communiqua même au chancelier deux versions du frontispice de son projet. Cette entreprise éditoriale ayant avorté pour des raisons inconnues, seules sont aujourd'hui connues les lettres de Röslin - celles de Mästlin sont apparemment perdues ${ }^{31}$.

Après avoir achevé ses études de médecine à Tübingen en 1569 et élargi ses connaissances dans les domaines de l'astrologie et de l'alchimie à Durlach (Baden) avec Samuel Eisenmenger et à Pforzheim avec le pharmacien Gröninger, Helisaeus Röslin s'était installé en 1572 en Alsace, en qualité de médecin personnel de Georg Hans I von Pfalz-Veldenz (1543-1592), comte palatin du Rhin. L'apparition de la nova de Cassiopée en novembre 1572, interprétée comme une étoile nouvelle dans la sphère des fixes, et les phénomènes extraordinaires qui s'ensuivirent jusqu'à la grande comète de 1577, ont été l'occasion de sa première publication : une Theoria nova cœlestium $\mu \varepsilon \tau \varepsilon \omega \varrho \omega v$ (Strasbourg, Bernard Jobin, 1578), où la nova de 1572 et la comète de 1577 sont comprises, en raison de leur caractère miraculeux, comme des signes eschatologiques de l'accomplissement de l'histoire du monde.

À cette époque, Röslin avait déjà quasiment achevé un imposant ouvrage manuscrit, intitulé Speculum et harmonia mundi : Das ist : Wellt Spiegel Erster Theil, dont il entreprit sans succès la publication dans les années suivantes, et dont la copie manuscrite demeure aujourd'hui conservée à la Württembergische Landesbibliothek de Stuttgart ${ }^{32}$. Le manuscrit fut imprimé à son insu en 1604, puis derechef en 1616 , l'année de sa mort ${ }^{33}$. Cette vaste étude contient la quasi-totalité des thèmes abordés par Röslin dans son œuvre postérieure, bien qu'il ne constitue formellement que le premier volet d'un ensemble plus vaste. Le Weltspiegel comprend en effet l'examen du cours de l'histoire, sacrée et profane, et compare les structures générales des différents règnes et époques pour déduire et dévoiler, à partir de leurs correspondances et des parallélismes découverts, les temps à venir. C'est donc plutôt la seconde partie, le Naturspiegel, comprenant le versant astronomique et cosmologique, qui faisait encore défaut, puisque la troisième partie (le Kirchenspiegel relatif à l'histoire de l'Église et aux prophéties eschatologiques) trouvait un premier exposé dans le Weltspiegel.

Si ce Naturspiegel trouve une présentation dans deux ouvrages publiés en 1597 (le susmentionné De opere Dei creationis et le Tractatus meteorastrologiphysicus) ${ }^{34}$, Röslin réalise une synthèse des Welt- et Kirchenspiegel dans un manuscrit anonyme rédigé en 1580 et conservé 


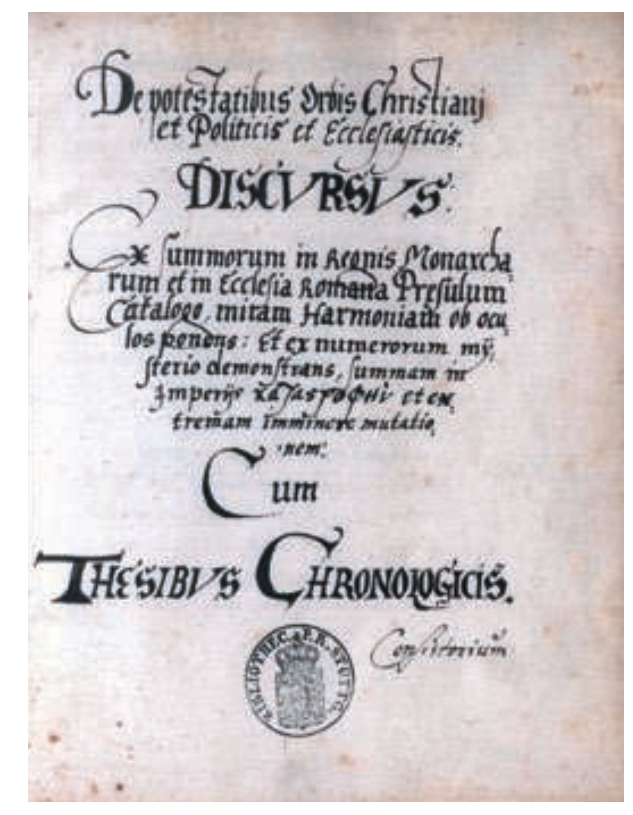

Page de titre du De potestatibus orbis christiani de Röslin (coll. Württembergische Landesbibliothek Stuttgart)

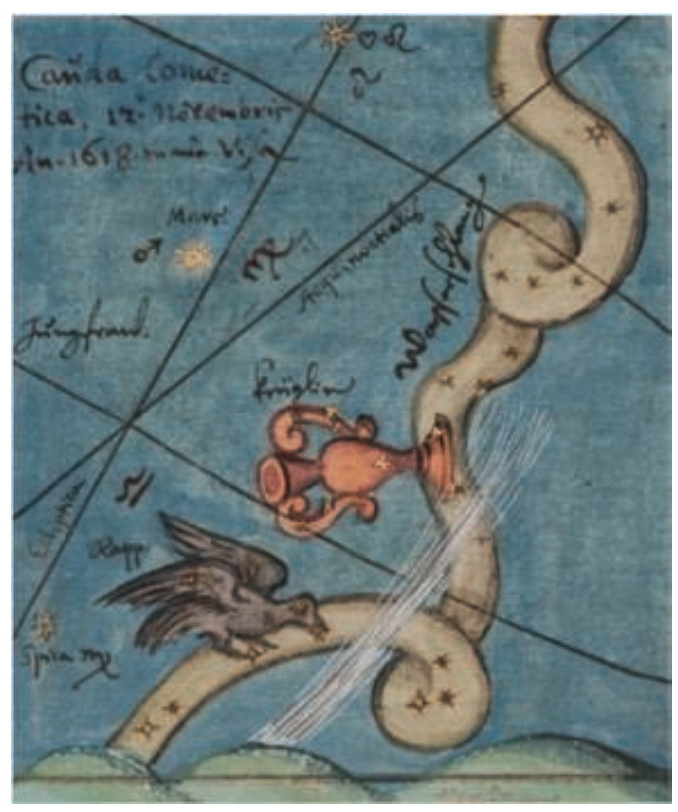

Wilhelm Schickhard, queue de la comète vue le 12 novembre 1618 (coll. Württembergische Landesbibliothek Stuttgart) lui aussi à la WLB sous le titre De potestatibus Orbis Christiani... ${ }^{35}$, dont nous avons montré ailleurs que Röslin est incontestablement l'auteur ${ }^{36}$.

Comme ce titre l'indique, Röslin était convaincu d'avoir découvert la clé de l'Histoire universelle, gouvernée par la providence divine manifeste dans la proportion des nombres des pouvoirs politiques et ecclésiastiques dans les trois périodes de l'Histoire (celles du "Vide ", de la "Loi " et de l'« Évangile " - deux mille ans chacune -, en référence à la célèbre prophétie dite de la Maison d'Élie).

Quand le pape Grégoire XIII promulgua en 1582 la réforme du calendrier julien, Röslin, sous le pseudonyme de Lambertus Floridus Plieninger ${ }^{37}$, ne tarda pas à réagir par une féroce diatribe contre cette réforme, dénoncée comme l'ultime effort de l'Antéchrist romain pour récupérer le pouvoir moral et politique perdu avec l'avènement de la Réforme protestante. Ce Kurtz Bedencken ${ }^{38}$ fut réimprimé l'année suivante par Michael Mästlin, alors professeur de mathématiques à Heidelberg, avec d'autres réactions protestantes, non moins virulentes, y compris celle de Mästlin lui-même, qui entamait ainsi un long combat avec le champion catholique de la réforme, le mathématicien jésuite Christophorus Clavius.

Röslin et Mästlin, en Alsace et dans le Wurtemberg, prêtaient beaucoup d'attention aux nouveautés célestes (novas et comètes) de l'époque, soit pour leurs implications cosmologiques, soit pour leurs significations astrologique et eschatologique - la dimension purement astrologique étant toutefois plutôt négligée par Mästlin. Parmi les manuscrits mästliniens conservés à la WLB, on trouve deux traités, inachevés, sur la nova de $1604^{39}$ et sur la grande comète de 1618 . Cette comète était la première qui pouvait être observée au télescope, et la coïncidence de son apparition avec le début de la guerre de Trente Ans suscita une foule de publications en Allemagne, parmi lesquelles le grand traité de Kepler De cometis libelli tres (Augsburg, $1619)^{40}$. Mais plusieurs traités inédits restent en attente d'être étudiés et édités. C'est le cas de celui de Mästlin ${ }^{41}$ et d'un autre, également conservé à la WLB, dont l'auteur est le plus jeune collègue de ce dernier à Tübingen, Wilhelm Schickard (1595-1635). Si le traité de Mästlin reste inachevé et présente deux rédactions différentes qui offrent de nombreuses difficultés de lecture et d'interprétation, le traité de Schickard, beaucoup plus étendu, est complet ${ }^{42}$.

Dédié au duc de Wurtemberg et agrémenté d'une somptueuse iconographie polychrome, il constitue un document à tous points de vue exceptionnel. Il est probable que Wilhelm Schickard obtint sa nomination comme professeur de langue hébraïque à l'Université de Tübingen en récompense de ce beau travail. La WLB donne libre accès à une reproduction de ce manuscrit ${ }^{43}$, qui attend encore un travail d'édition et de commentaire dans le cadre d'une étude sur les enjeux de la littérature cométaire entre 1572 et 1618 . 


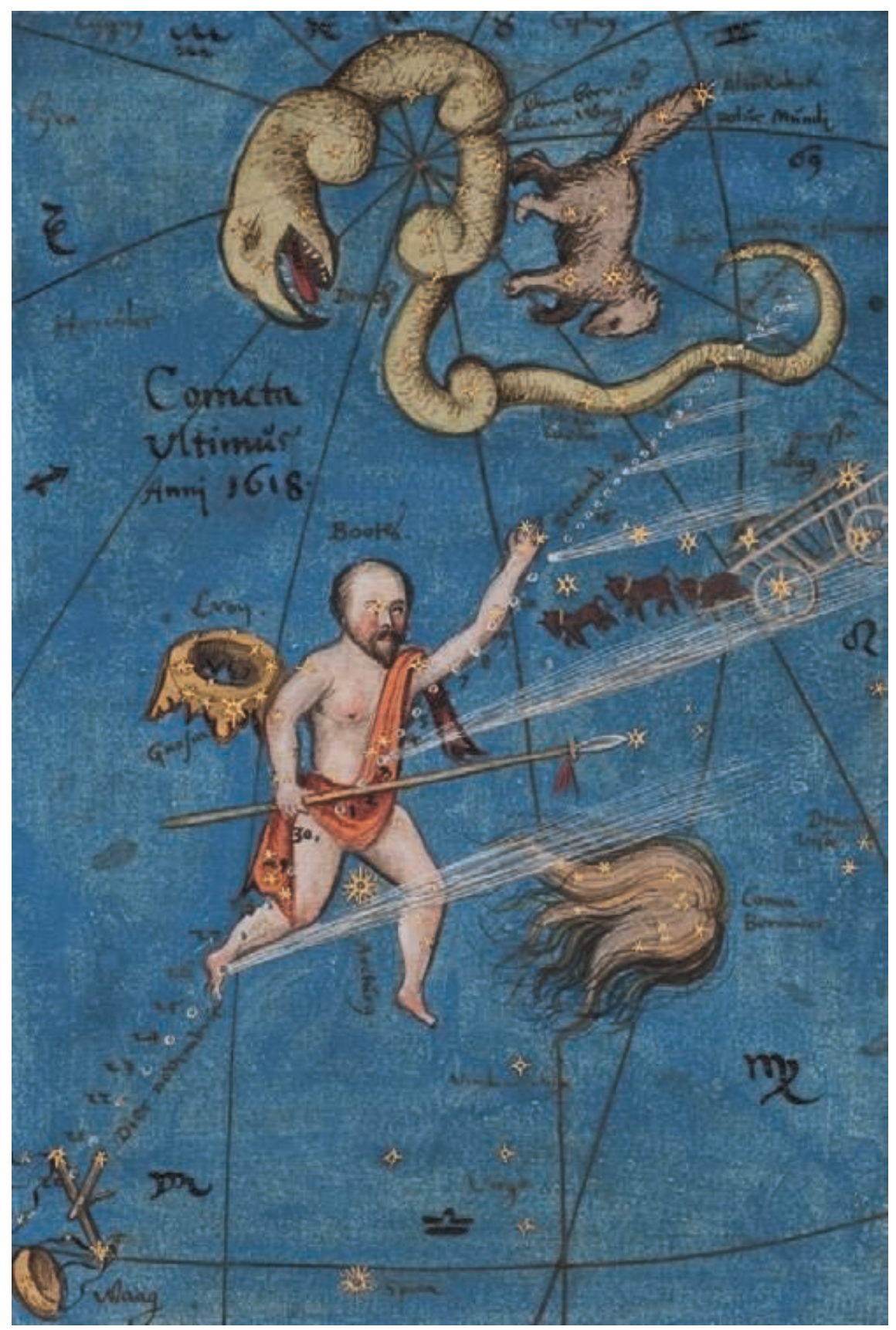

Wilhelm Schickhard, traité sur les comètes (coll. Württembergische Landesbibliothek Stuttgart) 
$\mathrm{Au}$ long de cette période, l'astronomie a connu une mutation profonde, qui n'est pas seulement liée, comme on l'a trop souvent dit, au choix des hypothèses géo- ou héliocentrique, mais aussi et d'abord au fait que les apparences célestes sont désormais considérées comme des événements : le passage des comètes $(1577,1585,1607,1618)$, l'apparition des nouvelles étoiles $(1572,1604)$, ou les premières observations des taches solaires (1611) sont autant d'événements dont la prise en compte amène l'astronomie mathématique à se transformer en une " physique céleste ", seule à même d'expliquer, à terme, ces phénomènes par l'interaction des forces qui les produisent. Ceci oblige les savants de l'époque, soucieux de retrouver une description fidèle des phénomènes considérés, à communiquer et à s'informer de manière beaucoup plus précise. Entre Copernic et Newton, la révolution scientifique n'aura donc pas été dissociable d'un développement particulièrement soutenu des métiers d'édition, de diffusion et de conservation de la littérature scientifique ancienne et moderne.

\section{Patrick J. Boner, Miguel A. Granada, Édouard Mehl}

\section{Notes}

1 - Owen Gingerich, An annotated Census of Copernicus' De Revolutionibus (Nuremberg, 1543, and Basel, 1566), Leiden-Boston-Köln, Brill, 2002

2 - Günther Oestmann, Die astronomische Uhr des Straßburger Münsters : Funktion und Bedeutung eines Kosmos-Modells des 16. Jahrhunderts, Verlag für Geschischte der Naturwissenschaften und der Technik, Stuttgart, 1993, Berlin, 2002

3 - Serafina Cuomo, Pappus of Alexandria and the Mathematics of Late Antiquity, Cambridge classical studies, 2000

4 - Outre Claude Ptolémée, Harmonica (manuscrit acquis par Dasypodius et offert au mathématicien et musicien David Wolkenstein en 1575 [voir infra n. 11], aujourd'hui conservé à la bibliothèque de l'Université d'Uppsala [ms gr. 45]), on peut notamment citer Aristoxène, Elementa harmonica, Alypius, Isagoge Musica, et les commentaires de Porphyre sur les Harmoniques de Ptolémée.

5- Sur Herwart von Hohenburg, voir infra, section suivante. C'est par son intermédiaire que Kepler a d'abord reçu, en 1600, les Harmoniques de Ptolémée dans la traduction d'Antoine Gogavino (1562). Quelques années plus tard, en 1607, insatisfait de cette traduction, Kepler se fait communiquer par Herwart une copie du manuscrit grec de Ptolémée, dont il entreprend une traduction partielle, et prend connaissance des commentaires anciens édités par Dasypodius. La connaissance de ces textes joue un rôle déterminant pour l'élaboration de l'ouvrage qui constitue la clef de voûte de son œuvre scientifique : l'Harmonice mundi (1619).

6 - Peter Barker, The Hypotyposes orbium cœlestium, 1568, in Nouveau Ciel, Nouvelle Terre. La révolution copernicienne dans l'Allemagne de la Réforme, 1530-1630, éd. par M. A. Granada et E. Mehl, Paris, Les Belles lettres, 2009, p. 85-108

7 - Matthias Bernegger $(1582-1640)$ est le correspondant strasbourgeois de Kepler (et propriétaire du portrait de Kepler conservé à la Fondation du Chapitre de Saint-Thomas). Bernegger s'est illustré en publiant, deux ans après le procès de Galilée (1633), une version latine du Dialogue sur les deux grands systèmes du monde (Systema cosmicum, [Strasbourg], David Hautt pour les Elzevir, 1635).

8 - Il s'agit du plus ancien et du plus authentique portrait de Copernic, réalisé par le peintre-graveur Tobias Stimmer, d'après un autoportait perdu. Sur Mästlin, voir infra, section 3.

9 - Cunrad Dasypodius à Andreas Düdith, 3 décembre 1571, lettre-préface aux Propositiones Logisticae Astronomicae Barlaami Monachi (Strasbourg, 1572), fol. F 2 sq. : « Nunc unicum hoc abs te Magnifice D. Sbardellate obnixe peto : ut doctiss. Virum D. Ioachimum Rheticum moneas sui officii et promissionis factae : \& ut ea de quibus mihi saepius scripsit, evulget " [Je ne vous demande maintenant instamment qu'une seule chose : de rappeler le très savant Maître Ioachim Rheticus à son devoir, et à la promesse qu'il a faite de montrer au public les choses dont il m'a souvent écrit].

10 - Voir les notices du Corpus Paracelsisticum, Bd. I, éd. W. Kühlmann et J. Telle, Tübingen, Niemeyer, 2001, p. 57-103 (sur Rheticus), et Bd. II, 2004, passim (sur Michael Toxites). On peut rappeler ici que Rheticus est un surnom, que Michael Schütz (Toxites) et Georg Ioachim Iserin (Rheticus) avaient partagé dans leur jeunesse.

11 - Ursus publie notamment à Strasbourg le Fundamentum Astronomicum (1588). Sur Ursus, ses rapports avec Conrad Dasypodius et son assistant David Wolkenstein (Nephelitus, 1534-1592), voir Dieter Launert, Nicolaus Reimers (Raimarus Ursus) : Günstling Rantzaus - Brahes Feind : Leben und Werk, München, Institut für Geschichte der Naturwissenschaften, 1999, p. 58-67; 195-197.

12 - Johann Georg Herwart von Hohenburg à Tycho Brahe, 17 septembre 1599, in Tycho Brahe Opera Omnia, éd. J. L. E. Dreyer [TBOO], 8, Copenhagen, 1925, 175.1-2

13 - Johann Georg Herwart von Hohenburg, Catalogus graecorum manuscriptorum codicum, Ingolstadt, 1602 
14- Sur Röslin, voir infra, section 3; sur la tolérance religieuse, voir Miguel A Granada, Helisaeus Röslin y la libertad de religión, in Anales del seminario de historia de la filosofía, 31 (2014), p. 69-88.

15 - Plusieurs visiteurs sont venus de l'Université d'Ingolstadt pour accéder à la bibliothèque de la cour. Comme Johann Appenzeller le rapporte à Herwart dans une lettre datée du 7 mai 1599, Balthasar Hagelius est venu à Munich pour y étudier les sources anciennes, en chaldéen et en hébreu. Appenzeller enseignait les mathématiques, et Hagelius la philosophie (J. Appenzeller à Herwart von Hohenburg, 7 mai 1599, Bayerische Staatsbibliothek, Cod. lat. 1607, p. 192-199). Dans une lettre suivante, où il remercie Herwart pour l'envoi de deux manuscrits, Appenzeller demande s'il serait envisageable d'en faire une traduction latine (J. Appenzeller à Herwart von Hohenburg, Bayerische Staatsbibliothek, Cod. lat. 1607, p. 338-341). Sur Appenzeller à l'Université d'Ingolstadt, voir Robert S. Westman, The Copernican Question: Prognostication, Skepticism, and Celestial Order, Berkeley, 2011, p. 312.

16-J. Appenzeller à Herwart von Hohenburg, 7 mai 1599, ibid., p. 192

17 - Sur les nombreuses tentatives pour cartographier et comprendre les mouvements de la lune, voir Chantal Grell (éd.), La lune aux XVII et XVIII siècles, Turnhout, 2013.

18 - J. Appenzeller à Herwart von Hohenburg, 7 mai 1599, ibid., p. 193

19 - Ibid., p. 194

20 - J. G. Herwart von Hohenburg à Tycho Brahe, 23 juillet 1599, TBOO, $8,158.5,158.7-10 ; 157.20-25 ; 157.34-36 ; 158.16-18$

21 - J. G. Herwart von Hohenburg à Tycho Brahe, 17 septembre 1599, TBOO, 8, 175.2-5. Sur le rôle d'Herwart vis-à-vis de Tycho, voir Adam J. Mosley, Bearing the Heavens: Tycho Brahe and the Astronomical Community of the Late Sixteenth Century, Cambridge, 2007, p. 173-174.

22 - Tycho Brahe à Herwart von Hohenburg, 16 novembre 1599, TBOO, 8, 202.19-20

23 - Ibid., 202.28-29, puis 203.8-11

24-Tycho Brahe à Herwart von Hohenburg, 31 août 1599, TBOO, 8, 160.8-14. Dans son échange avec Herwart, Tycho fit montre d'une grande érudition et de ses talents d'exégète. Pour d'autres exemples de cette émulation en milieu scientifique, voir Anthony Grafton, Defenders of the Text: The Traditions of Scholarship in an Age of Science, 1450-1800, Cambridge, MA, 1991.

25- Ibid., 16o.14-18; 23-25. Sur le développement de la cométologie aux $16^{\mathrm{e}}-17^{\mathrm{e}}$ siècles, voir Adam Mosley, The History and Historiography of Early Modern Comets, in Miguel A. Granada, Adam Mosley et Nicholas Jardine, Christoph Rothmann's Discourse on the Comet of 1585, Leiden, 2014, p. 282-325.

26 - Joseph Scaliger à Tycho Brahe, 10 mars 1600, TBOO, 8, 264.4-5, puis 263.40-41. Le poète qui fait l'objet de cette question suspicieuse est Caius Albinovanus Pedo, effectivement actif au tournant du $1^{\text {er }}$ siècle après J.-C., et avec qui Ovide s'entretenait notamment de sujets astrologiques (Pontiques, IV, 11), mais l'épisode évoqué ici ne figure pas dans les rares fragments conservés.

27 - Tycho Brahe à Herwart von Hohenburg, 28 août 16oo, TBOO, 8, 352.9-13

28-Sur les nombreux ouvrages que Kepler obtint par l'intermédiaire d'Herwart, voir Patrick J. Boner, Statesman and Scholar: Herwart von Hohenburg as Patron and Scholar in the Republic of Letters, in History of Science, 52 (2014), 1-23.

29-Voir M. A. Granada, Helisceus Roslin contre Raymarus Ursus après la publication $d u$ De astronomicis hypothesibus : ses lettres à Herwart von Hohenburg de 1597, in Omnia in uno : hommage à Alain-Philippe Segonds, textes réunis par Caroline Noirot et Nuccio Ordine, Paris, Les Belles lettres, 2012, p. 425-451.

30 - Voir Nicholas Jardine, Alain Philippe Segonds, La guerre des astronomes. La querelle au sujet de l'origine du système géo-héliocentrique à la fin du XVI siècle, Paris, Les Belles lettres, 2008 (3 vol.). Les Thèses strasbourgeoises d’Ursus (1588) sont rééditées dans M. A. Granada, Sfere solide et cielo fluido. Momenti del dibattito cosmologico nella seconda metà del Cinquecento, Milan, Guerini e Associati, 2002, p. 263-278.
31 - Les lettres de Röslin, conservées à la Württembergische Landesbibliothek (WLB) de Stuttgart (Cod. math. 4 14b, recueillant la correspondance de Mästlin), ont été partiellement publiées dans M. A. Granada, Sfere solide e cielo fluido, p. 279-294.

32 - Cod. theol. et philos., fol. 62

$33-\mathrm{H}$. Röslin, Speculum et harmonia mundi: Das ist: Weltspiegel mit Vergleichung der Monarchien und Welt-regimenten durch die Umbständen der Orter, Zeiten und Personen, auß Gottes Rähtschlag mit denselbigen, nach seinem Werck der Creation vnd Schöpffung fürgenommen, von Anfang der Welt biß zum End geführt [...], Frankfurt, J. C. Unckel, 1616

34-Tractatus meteorastrologiphysicus [...], Strasbourg, Bernhart Jobins Erben, 1597. Röslin lui-même le dit dans son manuscrit Ratio studiorum et operum meorum (Strasbourg, Archives de la Ville, Archives de St.-Thomas, 101, 4). Voir M. A. Granada, Helisaeus Röslin on the eve of the appearance of the nova of 1604 : his eschatological expectations and his intellectual career as recorded in the Ratio studiorum et operum meorum (1603-1604), in Sudhoffs Archiv, 90, 2006, p. 75-96.

35- Le titre exact en est : De potestatibus Orbis Christiani/ et Politicis et Ecclesiasticis./ DISCURSUS:/ Ex summorum in Regnis Monarcha-/ rum et in Ecclesia Romana Praesulum/Catalogo, miram Harmoniam ob ocu-/ los ponens:

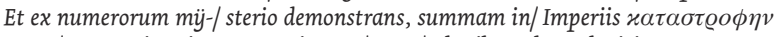
et ex-/tremam imminere mutationem:/ Cum/ Thesibus Chronologicis.

$36-$ M. A. Granada, Helisaeus Roeslin's Chronological Conception and a New Manuscript Source, in Early Science and Medicine, 18, 2013, p. 231-265

37 - Sous le pseudonyme de Lambertus Floridus Plieningerus, dont il se servira dans ses nombreux manuscrits théologiques en allemand, tous disparus à l'exception de la traduction latine du commentaire de 4 Esdras : Interpretatio Mystica et Vera in Quartum librum Esdrae Prophetae, traduction latine par Karl Widemann offerte au roi Jacques Ir d'Angleterre (British Library, MS Hologr. 2.D.XX). Sur ce manuscrit, voir M. A. Granada, Helisaeus Röslin : chronologie, astronomie, histoire... et prophétie (4 Esdras et l'avènement du royaume du Christ), sous presse dans Le Temps des astronomes. Astronomie, chronologie, histoire, du Moyen-Âge aux Lumières (éd. Édouard Mehl), Paris, Les Belles lettres, 2015.

38 - Kurtz Bedencken Von der Emendation dess Jahrs/ durch Bapst Gregorium der XIII. fürgenommen/ und von seinem Kalender [...], Strasbourg, Josiah Rihel, 1583

39 - M. Mästlin, Consideratio Astronomica inusitatae Novae et prodigiosae Stellae, superiori 1604 anno... exortae... ; voir M. A. Granada, Michael Maestlin and his unpublished treatise on the nova of 1604, in Journal for the History of Astronomy, XLV (2014), p. 91-122.

40-Voir M. Gindhart, Das Kometenjahr 1618: antikes und zeitgenössisches Wissen in der frühneuzeitlichen Kometenliteratur des deutschsprachigen Raumes, Wiesbaden, Dr. Ludwig Reichert Verlag, 2006

41 - M. Mästlin, Astronomischer Discurs von dem Cometen, so in Anno 1618. im [Monate] Novembris zu erscheinen angefangen und bis im [Monate] Februarii dis 1619 Jars am Himmel noch gesehen wirt.

42-Son titre exact est : Cometen Beschreibung In zwen underschidliche Partes abgetheilt, deren Erster Von denselbigen ins gemein: der Ander Von allen Insonderheit, sonderlich aber denen drë̈Jüngsten, In abgeloffenen $1618 \mathrm{Kahr}$ erschienen, aussführlich handelt.

43 - Cod. math. qt. 43 : http://digital.wlb-stuttgart.de/purl/bsz307044173 